\title{
Reduced order methods for nonlinear parametric problems with branching solutions
}

Federico Pichi ${ }^{1}$, Martin Wilfried Hess ${ }^{1}$,

Annalisa Quaini ${ }^{2}$, Gianluigi Rozza ${ }^{1}$

${ }^{1}$ SISSA mathLab, Trieste, Italy

${ }^{2}$ Math Department, University of Houston, TX, Usa

\section{Introduction}

The aim of this work is to show the applicability of the Reduced basis (RB) model reduction in nonlinear systems undergoing bifurcations. Bifurcation analysis, i.e. following the different bifurcating branches and determining the bifurcation point itself, is a complex computational task. Reduced Order Models (ROM) can potentially reduce the computational burden, enabling fast online evaluation of the solution for arbitrary parameter values.

\section{Application in elasticity: Von Kármán equations}

Let us consider an elastic, bi-dimensional and rectangular plate $\Omega=[0, L] \times[0,1]$ in its undeformed state, subject to a $\lambda$-parametrized external load acting on its edges. The displacement from its flat state and the Airy stress potential, respectively $u$ and $\phi$, satisfy the Von Kármán equations

$\left\{\begin{array}{ll}\Delta^{2} u=[\lambda h+\phi, u]+f, & \text { in } \Omega \\ \Delta^{2} \phi=-[u, u], & \text { in } \Omega\end{array} \quad \begin{cases}u=\Delta u=0, & \text { in } \partial \Omega \\ \phi=\Delta \phi=0, & \text { in } \partial \Omega\end{cases}\right.$

where $h$ and $f$ are the some given external forces acting on our plate, while

$$
\Delta^{2}:=\Delta \Delta=\left(\frac{\partial^{2}}{\partial x^{2}}+\frac{\partial^{2}}{\partial y^{2}}\right)^{2}, \quad[u, \phi]:=\frac{\partial^{2} u}{\partial x^{2}} \frac{\partial^{2} \phi}{\partial y^{2}}-2 \frac{\partial^{2} u}{\partial x \partial y} \frac{\partial^{2} \phi}{\partial x \partial y}+\frac{\partial^{2} u}{\partial y^{2}} \frac{\partial^{2} \phi}{\partial x^{2}}
$$

are the biharmonic operator in Cartesian coordinate and the bracket of Monge-Ampére respectively.

\section{Bifurcation problem and spectral analysis}

We represent the non-linear PDE with the parametrized mapping $G: V \times \mathcal{D} \rightarrow V^{\prime}$, so that the weak form of the Von Kármán system reads: given $\lambda \in \mathcal{D}$, find $X(\lambda) \doteq(u(\lambda), U(\lambda), \phi(\lambda), \Phi(\lambda)) \in V$ s.t.

$$
g(X(\lambda), Y ; \lambda) \doteq\langle G(X(\lambda) ; \lambda), Y\rangle=0, \quad \forall Y \in V .
$$
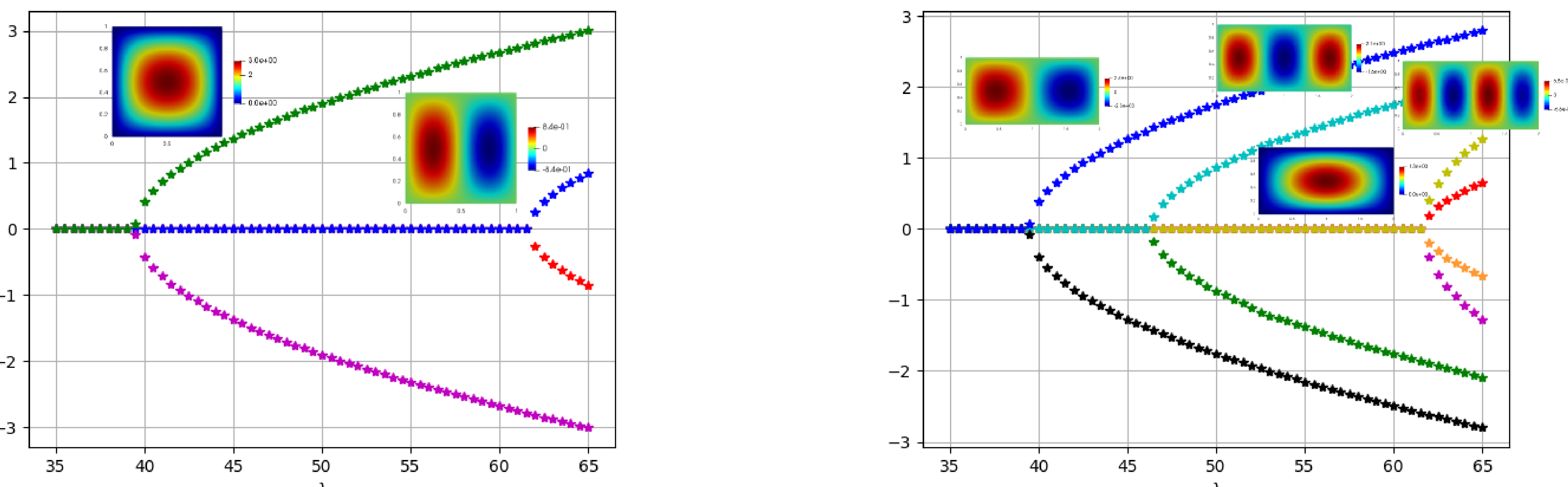

We say that $\lambda^{*} \in \mathbb{R}$ is a bifurcation point for $G: V \times \mathcal{D} \rightarrow V^{\prime}$, from the trivial solution, if there is a sequence $\left(X_{n}, \lambda_{n}\right) \in V \times \mathbb{R}$ with $X_{n} \neq 0$ and $G\left(X_{n}, \lambda_{n}\right)=0$ such that $\left(X_{n}, \lambda_{n}\right) \rightarrow\left(0, \lambda^{*}\right)$. A necessary condition for $\lambda^{*}$ to be a bifurcation point for $G$ is that $D_{X} G\left(0 ; \lambda^{*}\right)$ is not invertible. From the algebraic viewpoint the RB method reads: find $\delta \vec{X}_{N} \in \mathbb{R}^{N}$ such that

$$
\mathbb{J}_{N}\left(\vec{X}_{N}^{k}(\lambda) ; \lambda\right) \delta \vec{X}_{N}=G_{N}\left(\vec{X}_{N}^{k}(\lambda) ; \lambda\right), \quad \text { and } \quad X_{N}^{k+1}=X_{N}^{k}-\delta X_{N}
$$

Moreover, the linearization around the null solution provides us the following eigenproblem, where the parametrized eigenvalue $\sigma_{\lambda} \in \mathbb{R}$, crossing the imaginary axis, induces the bifurcation

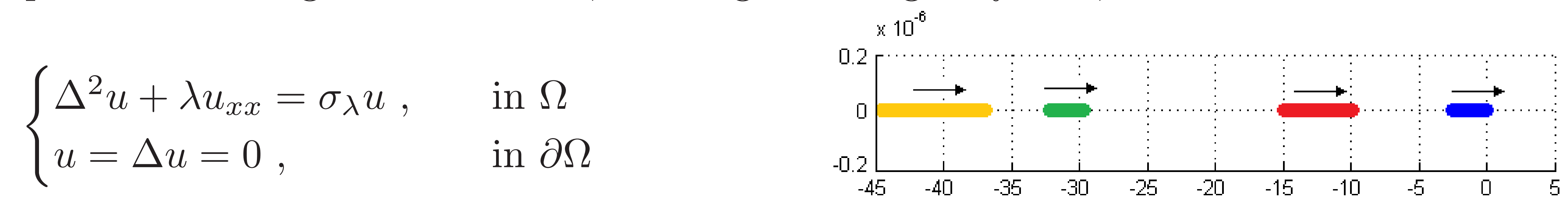

The Von Kármán reduced framework is continuing in collaboration with A. Patera (MIT).

\section{Application in CFD \\ We consider the incompressible Navier-Stokes equations in $\Omega_{T}=\Omega \times(0, T]$

$$
\begin{cases}\frac{\partial \boldsymbol{u}}{\partial t}+(\boldsymbol{u} \cdot \nabla \boldsymbol{u})-\nu \Delta \boldsymbol{u}+\nabla p=\mathbf{0} & \text { in } \Omega_{T} \\ \nabla \cdot \boldsymbol{u}=0 & \text { in } \Omega_{T}\end{cases}
$$

discretized with the spectral element method We focus on a jet flow entering a sudden expansion channel. The system undergoes a supercritical pitchfork bifurcation with increasing Reynolds-number $R e=\frac{2 U w}{\nu}$, that characterizes the flow regime.

For sufficiently small value of $R e$, a steady symmetric jet is observed. Above a certain critical Reynolds number, two stable solutions emerge, with the flow attaching itself to the top and lower boundary. This "wall-hugging" is a wellknown phenomenon called the Coanda-effect.

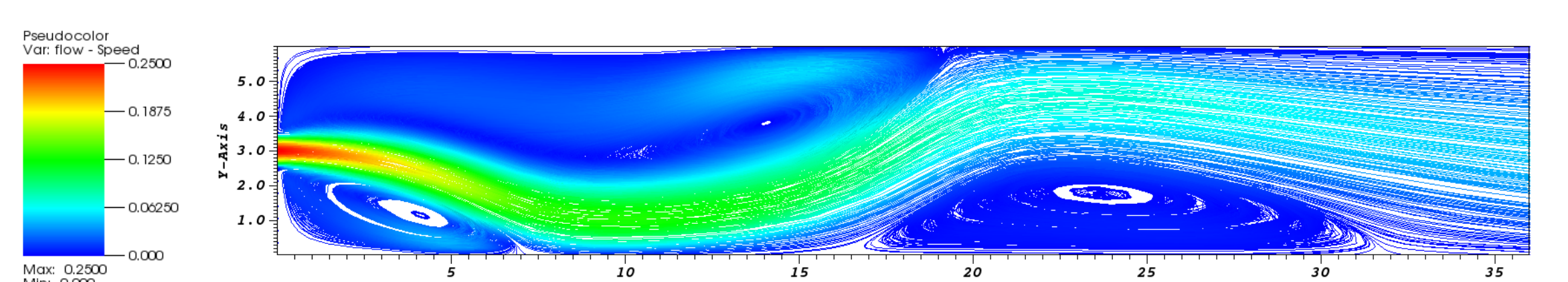

Channel flow solution at Reynolds number 100.

\section{Reduced Order Modeling}

To allow for bifurcation analysis of complex problems, reduced order modeling can play an important role. A RB space is generated from snapshot solutions to recover the bifurcation diagram and accurately find the bifurcation point.

Bifurcation diagram over Reynolds number 5 to 100. Plot of the velocity in vertical direction at the point $(x, y)=(1,3)$.

Localized bases, each corresponding to different bifurcation branches, are under current investigation.

This work is continuing in collaboration with $\mathrm{M}$ Gunzburger (FSU) and A. Alla (PUC-Rio).

\section{References}

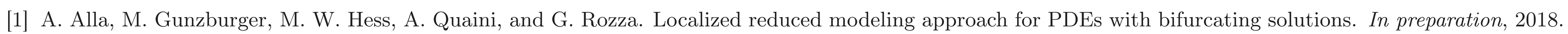

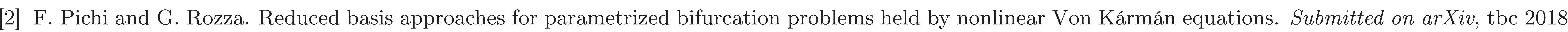

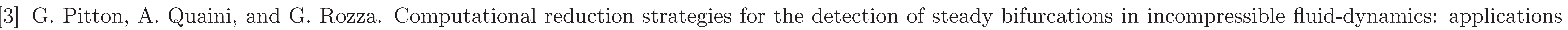
to Coanda effect in cardiology. Journal of Computational Physics, 344:534-557, 2017

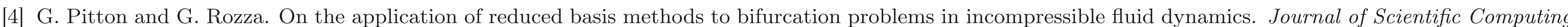
73(1):157-177, 2017

\section{Acknowledgements}

